\title{
Comparison of clinical and magnetic resonance imaging findings of triple-negative breast cancer with non-triple-negative tumours
}

\author{
Duygu İmre Yetkin ${ }^{1 A, B, C, D, E, F, G}$, Meltem Gulsun Akpınar ${ }^{2 A, B, D, E}$, Gamze Durhan ${ }^{2 A, B, E}$, Figen Basaran Demirkazik²A,B \\ 'Midyat State of Hospital, Midyat/Mardin, Turkey \\ ${ }^{2}$ Hacettepe University Faculty of Medicine, Ankara, Turkey
}

\section{Abstract}

Purpose: Triple-negative breast cancer (TNBC) has some distinctive features. The aim of the study was to compare clinical and breast magnetic resonance imaging (MRI) findings of TNBC with non-triple-negative breast cancer (nTNBC) in molecular subtypes such as ADC (apparent diffusion coefficient) values, T2-weighted (T2W) image intensity, shape, margin, lymph node involvement, grade, multifocality, multicentricity, bilaterality, and enhancement pattern differences between tumour subtypes.

Material and methods: A total of 141 patients who underwent breast biopsy at our institution between January 2010 and June 2018 were included in this study. Patients were divided into molecular subtypes according to hormone receptor status, and Ki-67 index. Tumour grade, enhancement patterns, age, lymph node involvement, ADC values, breast imaging reporting and data system (BI-RADS) category, bilaterality, multifocality, multicentricity, margin, shape, and T2W image intensity were evaluated for these subtypes.

Results: ADC values were higher in triple-negative tumours than in luminal A and luminal B tumours ( $p=0.010$ and $p=0.002$, respectively). Circumscribed margin, type 2 enhancement curve, and rim enhancement were significantly higher in triple-negative tumours $(p<0.001)$. No significant difference was found between the groups in terms of other MRI findings including bilaterality, multifocality, multicentricity, shape, and T2W image intensity $(p>0.05)$.

Conclusions: ADC values, circumscribed margin, and rim enhancement can provide important information about the tumour's biological behaviour and the course of the disease.

Key words: ADC, breast MRI, triple-negative tumours.

\section{Introduction}

Breast cancer is the most commonly seen type of cancer in women and a highly heterogeneous cancer with various morphological features, clinical courses, and treatment responses [1]. This diversity leads to the need for a standardized management in the diagnosis and treatment of the disease [2,3]. Immunohistochemical markers, besides the classical parameters such as tumour size, grade, and lymph node involvement, play a considerable role in subtyping the disease, determining prognosis, and selecting the treatment protocol [3-5]. According to the immunohistoche- mical markers, breast cancer is divided into several subtypes [6]. Triple-negative breast cancer (TNBC) accounts for about $15 \%$ of all breast cancer cases and is associated with a poor prognosis and a high ratio of distant recurrence [7-9]. Diagnosis of TNBC and distinction from other subgroups is essential for the treatment to be chosen. However up to $20 \%$ of immunohistochemical determinations of estrogen receptors (ER) and progesterone receptors (PR) testing may be inaccurate (false negative or positive). The reason for this difference can be due to variations in pre-analytic variables, positive threshold values, and evaluation criteria [10]. The presence of the features of TNBC

Correspondence address:

Duygu Imre Yetkin, Midyat State of Hospital, Midyat/Mardin, Turkey, e-mail: duyguimre0831@gmail.com

Authors' contribution:

A Study design · B Data collection · C Statistical analysis · D Data interpretation - E Manuscript preparation · F Literature search · G Funds collection 
in magnetic resonance imaging (MRI) distinguishing it from non-triple-negative breast cancer (nTNBC) may be cautionary in repetition of pathological specimen assessment if required. This can lead to significant differences in patient management.

Routinely, mammography and breast ultrasound are used in the evaluation of breast cancer. Breast MRI is used to exclude breast cancer or diseases requiring immediate treatment in symptomatic patients, preoperative staging, and treatment selection in patients with known malignancy (surgical or medical treatment, etc.). Breast MRI has the highest sensitivity (83-99\%) in detecting breast cancer among imaging modalities [11]. As well as for diagnosis of breast cancer, MRI findings may differentiate breast cancer subtypes.

In this study, we aimed to retrospectively evaluate the differences between TNBC and nTNBC in terms of MRI findings and clinical features. Lesions were classified according to immunohistochemical status. In addition, we aimed to evaluate parameters such as T2W image intensity, margin, size, lymph node involvement, multifocality, multicentricity, contrast enhancement patterns, and their relevance within molecular subtypes.

\section{Material and methods}

\section{Patients}

Approval for the study was granted by Hacettepe University Faculty of Medicine's Ethics Commission. Informed consent was not required because of the retrospective nature of the study. 141 of 2546 women who had a biopsyproven, $>5 \mathrm{~mm}$ mass forming invasive breast cancer at our institution between January 2010 and June 2018 were included in this study. The age of the participants was over 18 years. Lesions which were benign, compatible with metastasis or malignant phyllodes tumour, less than $5 \mathrm{~mm}$ in size or non-mass forming lesions, patients under 18 years of age, patients who underwent chemotherapy and/or surgery before breast MRI, and patients without diffusion-weighted imaging (DWI) and apparent diffusion coefficient (ADC) maps were excluded.

\section{Imaging}

The MRI scans were acquired with the patient in prone position using a 1.5-Tesla MRI scanner (Signa HD, GE Medical Systems, USA) with a 4-channel phased array breast coil. The imaging protocol included the following sequences: axial T2W fat saturated (TR/TE 5100/90 ms, slice thickness $[\mathrm{ST}]=2 \mathrm{~mm}$, flip angle $[\mathrm{FA}] 90^{\circ}$, matrix $256 \times 256$ ), axial echo-planar DWI (TR/TE 2500/72, $\mathrm{ST}=3 \mathrm{~mm}, \mathrm{FA}=90^{\circ}$, matrix $256 \times 256$, diffusion gradient with $b$ values of 0 and $1000 \mathrm{~s} / \mathrm{mm}^{2}$ ), and axial T1-weighted fast spin echo pre-contrast MR images (TR/TE 4.3/ $2.1 \mathrm{~ms}, \mathrm{ST}=2 \mathrm{~mm}, \mathrm{FA}=90^{\circ}$, matrix $\left.512 \times 512\right)$. Dy- namic breast examination was performed after the injection of intravenous contrast material (Dotarem, Guerbet, Roissy, France) through the antecubital vein at a dose of $0.1 \mathrm{mmol} / \mathrm{kg}$ using a power injector (Medrad, Bayer HealthCare, Netherlands). After pre-contrast T1-weighted images, the following 5 axial T1-weighted post-contrast dynamic sequences (TR/TE 4.5/2.1 $\mathrm{ms}, \mathrm{ST}=2 \mathrm{~mm}, \mathrm{FA}=10^{\circ}$, matrix $512 \times 512$ ) were obtained at intervals of $90 \mathrm{~s}$. Patients were evaluated retrospectively by consensus of 2 radiologists. ADC values of the patients were measured by free hand region of interest (ROI) (Figures 1 and 2). Areas of haemorrhage and necrosis were not included in the ROI. In cases of bilateral and multifocal lesions, biopsy performed lesion was selected to measure the ADC value. If the biopsy was taken from multiple lesions, the mean of these lesions was calculated. Age, pathologic diagnosis of lesions, tumour grade, lymph node involvement, morphological characteristics (margin, shape, T2 intensity), contrast enhancement kinetics (type 1, 2, 3), BI-RADS score, rim enhancement, multifocality, multicentricity, and bilaterality were recorded and evaluated.

\section{Pathological examination}

The pathological examination reports were reviewed to determine histopathological type, tumour grade, and immunohistochemical findings including ER, PR, and HER 2 status. Tumours that were ER positive, HER 2 negative, and with Ki $67<14 \%$ were defined as luminal A; ER positive, HER 2 positive, and Ki $67>14 \%$ were defined as luminal B; ER negative and HER 2 positive were defined as Her 2-enriched type; ER, PR, and HER 2 negative were defined as triple negative. The threshold value for ER and PR positivity was $1 \%$, for HER 2 positivity was either 3 positive or 2 positive, and the number of HER 2 gene amplifications in chromosome 17 by fluorescence in situ hybridization (FISH) was more than 2.2 [6]. According to ER, PR, HER2, and ki-67 status the lesions were divided into molecular subtypes (luminal A, luminal B, Her 2-enriched, and triple negative). The parameters described above were evaluated for each patient.

\section{Statistical analysis}

The research data were uploaded to the computer via SPSS for Windows 22.0 (SPSS Inc., Chicago, IL) and evaluated. Descriptive statistics were presented as mean \pm standard deviation (minimum-maximum), frequency distribution, and percentage. Pearson's $\chi^{2}$ test was used to evaluate categorical variables. The suitability of the variables to normal distribution was examined using visual (histogram and probability graphs) and analytical methods (KolmogorovSmirnov/Shapiro-Wilk test). For the variables that were not found to fit the normal distribution, the Mann-Whitney $U$ test was used for statistical significance between 2 independent groups; for 3 and more independent groups 

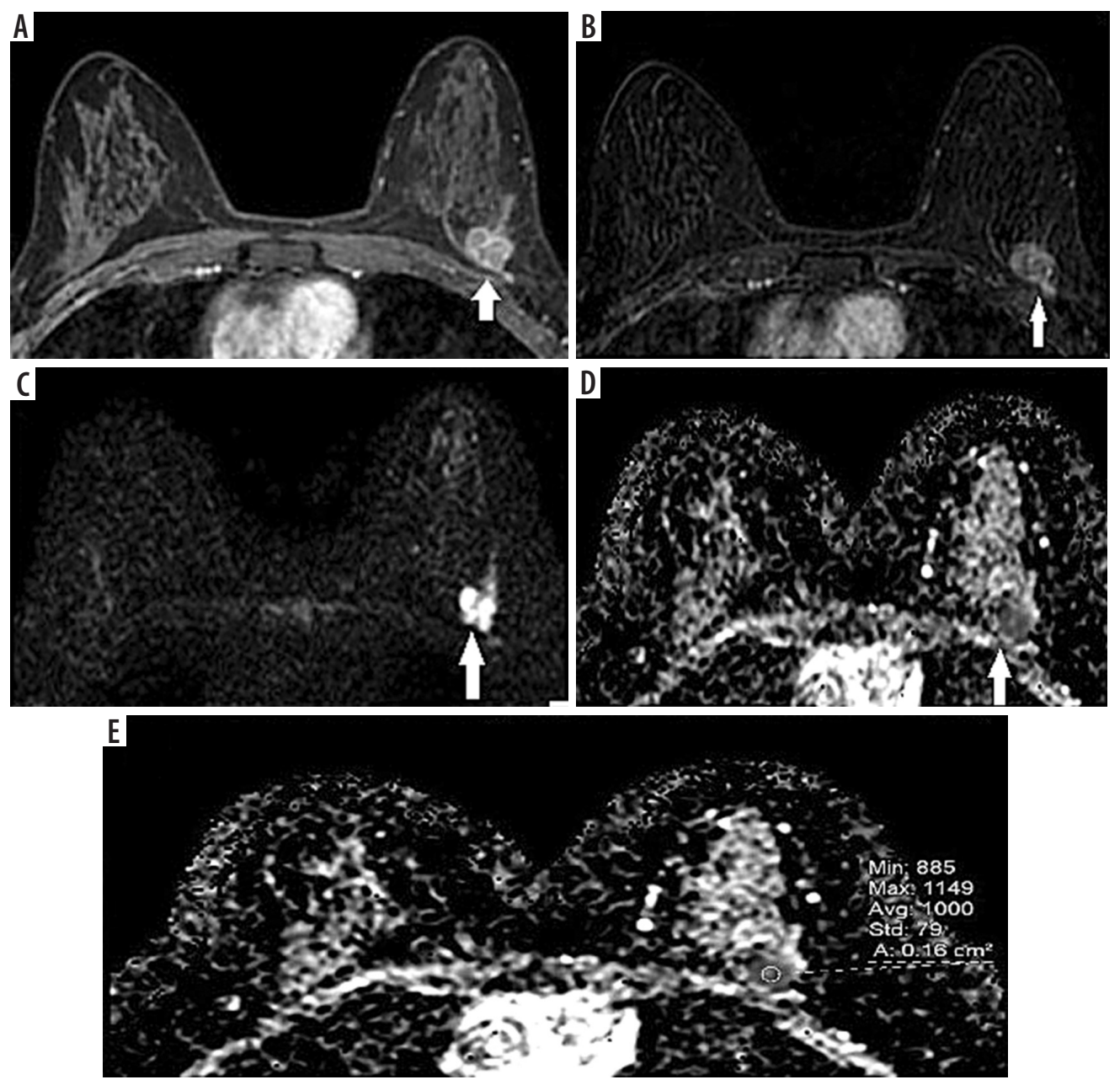

Figure 1. A 55-year-old woman with a mass on her left breast. A) Post-contrast with 4-minute delay T1W fat-sat image shows rim-enhancing lesion (white arrows). B) Subtraction images. C) DWI. D-E) ADC map $\left(1000 \times 10^{-3} \mathrm{~mm}^{2} / \mathrm{s}\right)$ images shows restriction. The pathology result of BI-RADS 5 mass, showing type 3 enhancement curve was ER-, PR-, HER2-, invasive ductal carcinoma grade 3, ki67 40\%

the Kruskal-Wallis test was used. When a significant difference was detected between 3 and more independent groups, Bonferroni correction was applied in post-hoc binary comparisons regarding the source of the difference. Statistical significance level was accepted as $p<0.05$.

\section{Results}

\section{Clinical and histopathological findings}

The data of 141 patients were analysed. The mean age was $51.4 \pm 10.1(\min 29 ; \max 75)$ years. $18.4 \%(n=26)$ of the patients were triple negative, $48.9 \%(n=69)$ were luminal B, $22.7 \%(n=32)$ were luminal $\mathrm{A}$, and $11.3 \%(n=16)$ were Her 2-enriched types. $70.9 \%$ of the patients were ER+, $63.1 \%$ were PR+, and $68.8 \%$ were HER 2-. Lymph node involvement was present in $56 \%$ of the patients.

Invasive ductal carcinoma was present in $85.1 \%$ of the patients, and $88.3 \%$ of them had unspecified type of invasive ductal carcinoma (IDC NOS). Mixed infiltrative type carcinoma was detected in $10 \%$ of the patients, $79 \%$ of which were NOS IDC and lobular carcinoma. Invasive lobular carcinoma was observed in $5 \%$ of patients.
MRI features and molecular findings of lesions according to histological grades are shown in detail in Table 1. Lymph node involvement was higher in patients with grade 3 tumours compared to grade 1 and 2 . The percentage of grade 3 in triple negative tumours was significantly higher than grade 1 and 2 . ER and PR positivity were higher, and HER2 positivity was lower in grade 1 tumours compared to grade 2 and $3(p<0.05)$.

The ratio of grade 1 tumour was higher in luminal A tumours and lower in luminal B tumours $(p<0.05)$. Axillary lymph node involvement was higher in Her 2-enriched tumours compared to other molecular subtypes and lower in luminal A tumours $(p<0.001)$.

Neither molecular subtypes nor tumour grades showed any statistically significant difference according to patient's age.

\section{Magnetic resonance imaging findings}

The average size of the lesions was $40.1 \times 28.6 \mathrm{~mm}$. $33.3 \%$ of the lesions were multifocal, $19.1 \%$ were multicentric, and $1.4 \%$ were bilateral. The margin of the lesions was spiculated in $50.4 \%$, irregular in $48.2 \%$, and circumscribed 

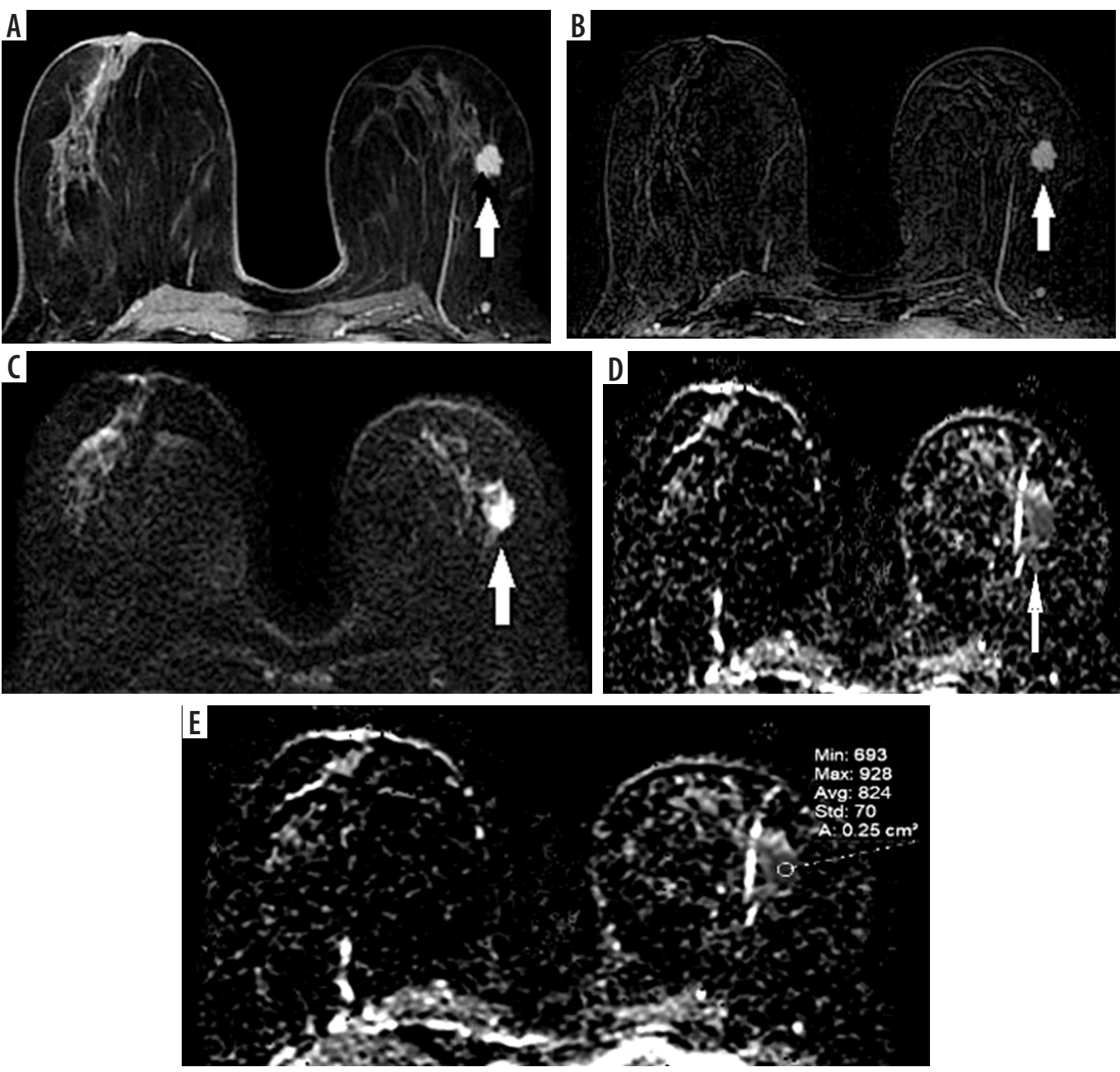

Figure 2. 56-year-old woman with a mass on her left breast. A) Post-contrast with 4-minute delay $\mathrm{T} 1 \mathrm{~W}$ fat-sat image shows homogenous enhancing lesion (white arrows). B) Subtraction images. C) DWI. D-E) ADC map $\left(824 \times 10^{-3} \mathrm{~mm}^{2} / \mathrm{s}\right)$ images shows restriction. The pathology result of BI-RADS 5 mass, showing type 3 enhancement curve was ER+, PR+, HER2-, invasive ductal carcinoma grade 1, ki67 5-7\%

in $1.4 \%$ of the cases. The shape of the lesions was irregular in $89.4 \%$, oval in $8.5 \%$, and round in $2.1 \%$ of the cases. Irregular margin was found most frequent in Her 2-enriched tumours, spiculated margin was most common in luminal B tumours, and circumscribed margin was most common in triple negative tumours $(p=0.027)$. On T2W images, $44 \%$ of the lesions were isointense, $28.3 \%$ were hyperintense, and $27.7 \%$ were hypointense. The mean ADC value was $1.138 \times 10^{-3} \mathrm{~mm}^{2} / \mathrm{s} .73 .8 \%$ of the lesions revealed type 3 , $21.2 \%$ type 2 , and $5 \%$ type 1 enhancement patterns. According to the BI-RADS classification, $58.2 \%$ of the lesions were classified as BI-RADS 5, 34.8\% as BI-RADS 6, and 7\% as BI-RADS 4.

ADC values of lesions according to molecular subtypes are shown detailed in Table 2 . The ADC value of triple negative tumours was higher compared to luminal A and luminal B tumours ( $p=0.010$ and $p=0.002$, respectively).

Rim enhancement was present in $17.7 \%$ of patients. It was most common in triple negative tumours compared to other molecular subtypes $(p<0.01)$. Type 3 enhancement curve was highest in luminal B tumours, type 2 was highest in triple negative tumours, and type 1 was highest in luminal A tumours $(p=0.018)$.

Type 2 and 3 enhancement curves were higher in grade 3 tumours, and type 1 enhancement was higher in grade 1 tumours $(p=0.029)$. No statistically significant difference was found between ADC values, rim enhancement, multifocality, multicentricity, and tumour grade (Table 1).

There was no statistically significant difference found between molecular subtypes in terms of T2 intensity, shape, BI-RADS category, multifocality, and multicentricity $(p>0.05$ for all) (Table 3$)$.

\section{Discussion}

The most important findings in this study were higher ratio of ADC values, rim enhancement pattern, type 2 enhancement curve, and circumscribed margin features in triple negative tumours compared to other molecular subtypes. On the other hand, T2 intensity, shape, BI-RADS category, multifocality, and multicentricity did not show any significant difference in triple negative lesions. 
Table 1. The distribution of age, lymph node involvement, side of the mass, hormone receptor status, molecular subgroups, and magnetic resonance imaging features of lesions according to tumour grade

\begin{tabular}{|c|c|c|c|c|}
\hline Parameter & Grade I $(n=13)$ & Grade II $(n=60)$ & Grade III ( $n=68)$ & $p^{\mathrm{a}}$ \\
\hline Age (year) & $50.2 \pm 7.3(42-67)$ & $52.6 \pm 10.8(34-75)$ & $50.5 \pm 9.8(29-70)$ & 0.754 \\
\hline Lymph node involvement, $n$ (\%) & $4(30.8)$ & $26(43.3)$ & $49(72.1)$ & 0.001 \\
\hline Bilaterality, $n(\%)$ & 0 & $2(3.3)$ & 0 & 0.531 \\
\hline $\mathrm{ER}(+), n(\%)$ & $13(100)$ & $51(85.0)$ & $36(52.9)$ & $<0.001$ \\
\hline $\mathrm{PR}(+), n(\%)$ & $13(100)$ & $42(70.0)$ & $34(50.0)$ & 0.001 \\
\hline Her2Neu(+), $n(\%)$ & 0 & $20(33.3)$ & $24(35.3)$ & 0.038 \\
\hline Triple negative, $n(\%)$ & 0 & $5(8.3)$ & $19(27.9)$ & 0.003 \\
\hline Her2 enriched, $n(\%)$ & 0 & $4(6.7)$ & $12(17.6)$ & 0.059 \\
\hline Luminal $\mathrm{A}, n(\%)$ & $12(92.3)$ & $20(33.3)$ & 0 & $<0.001$ \\
\hline Luminal $B, n(\%)$ & $1(7.7)$ & $31(51.7)$ & $37(54.4)$ & 0.007 \\
\hline \multicolumn{5}{|l|}{ Enhancement pattern, $n(\%)$} \\
\hline Type 1 & $3(23.1)$ & $3(5.0)$ & $1(1.5)$ & \multirow[t]{3}{*}{0.029} \\
\hline Type 2 & $2(15.4)$ & $13(21.7)$ & $15(22.1)$ & \\
\hline Type 3 & $8(61.5)$ & $44(73.3)$ & $52(76.4)$ & \\
\hline Rim enhancement, $n(\%)$ & $1(7.7)$ & $10(16.7)$ & $14(20.6)$ & 0.515 \\
\hline Multifocality, $n(\%)$ & $6(46.2)$ & $20(33.3)$ & $21(30.9)$ & 0.564 \\
\hline Multicentricity, $n(\%)$ & $2(15.4)$ & $7(11.7)$ & $18(26.5)$ & 0.098 \\
\hline $\operatorname{ADC}\left(\times 10^{-3} \mathrm{~mm}^{2} / \mathrm{s}\right)$, mean $\pm S D($ minimum-maximum) & $\begin{array}{l}1.139 \pm 0.228 \\
(0.821-1.793)\end{array}$ & $\begin{array}{l}1.135 \pm 0.262 \\
(0.781-2.391)\end{array}$ & $\begin{array}{l}1.141 \pm 0.258 \\
(0.542-1.676)\end{array}$ & 0.893 \\
\hline
\end{tabular}

MRI - magnetic resonance imaging, $A D C$ - apparent diffusion coefficient. ${ }^{2}$ Kruskal-Wallis test

Table 2. Molecular subtypes and apparent diffusion coefficient (ADC) correlation

\begin{tabular}{|l|c|c|}
\hline $\begin{array}{l}\text { Molecular subtype } \\
n / \text { mean } \pm \text { SD (min-max) }\end{array}$ & $\begin{array}{c}\text { Molecular subtype } \\
n / \text { mean } \pm \text { SD (min-max) }\end{array}$ & $p$-value \\
\hline Triple $(-)^{e}$ & Her-2 enriched & 0.145 \\
$24 / 1.287 \pm 0.271(0.878-1.870)$ & $16 / 1.152 \pm 0.216(0.871-1.624)$ & 0.010 \\
\hline $\begin{array}{l}\text { Triple }(-) \\
24 / 1.287 \pm 0.271(0.878-1.870)\end{array}$ & Luminal A & 0.002 \\
\hline Triple $(-)$ & $32 / 1.098 \pm 0.189(0.821-1.793)$ & 0.454 \\
$24 / 1.287 \pm 0.271(0.878-1.870)$ & Luminal B & \\
\hline Her-2 enriched & $69 / 1.102 \pm 0.270(0.542-2.391)$ & 0.345 \\
$16 / 1.152 \pm 0.216(0.871-1.624)$ & Luminal A & \\
\hline Her-2 enriched & $32 / 1.098 \pm 0.189(0.821-1.793)$ & 0.877 \\
$16 / 1.152 \pm 0.216(0.871-1.624)$ & Luminal B & \\
\hline Luminal A & $69 / 1.102 \pm 0.270(0.542-2.391)$ & Luminal B \\
$32 / 1.098 \pm 0.189(0.821-1.793)$ & $69 / 1.102 \pm 0.270(0.542-2.391)$ & \\
\hline
\end{tabular}

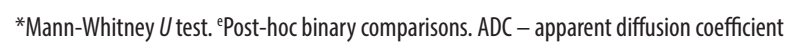

TNBC constitutes a small portion of all breast cancers, approximately $15-20 \%$; however, it is one of the major causes of breast cancer-related deaths due to the inability to use targeted therapy [12]. Knowing the imaging characteristics of triple negative tumours allows us to predict the biological behaviour of the tumour. In patients with imaging findings suggest a triple negative tumour and in whose pathological report is different from imaging findings, MRI features may play an important role in the changing treatment plan of the patients by enabling the re-evaluation of the pathological specimen.

In our study, the ADC value was higher in triple negative tumours than in luminal A and luminal B tumours. Xie et al. found that the ADC value of triple negative tumours was higher than that of luminal A tumours; however, no difference was found between luminal B and tri- 
Table 3. Comparison of morphological features and enhancement patterns between molecular subtypes

\begin{tabular}{|c|c|c|c|c|c|}
\hline Parameter & $\begin{array}{l}\text { Triple negative } \\
\qquad \begin{array}{c}(n=24) \\
n(\%)\end{array}\end{array}$ & $\begin{array}{l}\text { Her-2 enriched } \\
\qquad(n=16) \\
n(\%)\end{array}$ & $\begin{array}{c}\text { Luminal A } \\
\begin{array}{c}(n=32) \\
n(\%)\end{array}\end{array}$ & $\begin{array}{c}\text { Luminal B } \\
\qquad \begin{array}{c}(n=69) \\
n(\%)\end{array}\end{array}$ & $p^{\mathrm{a}}$ \\
\hline \multicolumn{6}{|c|}{ Enhancement pattern, $n(\%)$} \\
\hline Type 1 & $1(4.2)$ & 0 & $5(15.6)$ & $1(1.4)$ & \multirow[t]{3}{*}{0.018} \\
\hline Type 2 & $7(29.2)$ & $4(25.0)$ & $9(28.1)$ & $10(14.5)$ & \\
\hline Type 3 & $16(66.7)$ & $12(75.0)$ & $18(56.3)$ & $58(84.1)$ & \\
\hline Rim enhancement & $11(45.8)$ & $6(37.5)$ & $2(6.3)$ & $6(8.7)$ & $<0.001$ \\
\hline Multifocality & $10(41.7)$ & $7(43.8)$ & $12(37.5)$ & $18(26.1)$ & 0.332 \\
\hline Multicentricity & $7(29.2)$ & $5(31.3)$ & $2(6.3)$ & $13(18.8)$ & 0.089 \\
\hline \multicolumn{6}{|l|}{ T2 intensity } \\
\hline Isointense & $9(37.5)$ & $6(37.5)$ & $19(59.4)$ & 28 (40.6) & \multirow[t]{3}{*}{0.159} \\
\hline Hyperintense & $11(45.8)$ & $6(37.5)$ & $5(15.6)$ & $18(26.1)$ & \\
\hline Hypointense & $4(16.7)$ & $4(25.0)$ & $8(25.0)$ & $23(33.3)$ & \\
\hline \multicolumn{6}{|l|}{ Margin } \\
\hline \multicolumn{5}{|l|}{ Non-circumscribed } & \multirow[t]{4}{*}{0.027} \\
\hline - Spiculated & $8(33.3)$ & $6(37.5)$ & $17(53.1)$ & $40(58.0)$ & \\
\hline - Irregular & $14(58.3)$ & $10(62.5)$ & $15(46.9)$ & $29(42.0)$ & \\
\hline Circumscribed & $2(8.3)$ & 0 & 0 & 0 & \\
\hline \multicolumn{6}{|l|}{ Shape } \\
\hline Irregular & $20(83.3)$ & $13(81.3)$ & $31(96.9)$ & $62(89.9)$ & \multirow[t]{3}{*}{0.453} \\
\hline Oval & $3(12.5)$ & $3(18.8)$ & $1(3.1)$ & $5(7.2)$ & \\
\hline Round & $1(4.2)$ & 0 & 0 & $2(2.9)$ & \\
\hline \multicolumn{5}{|l|}{ BI-RADS } & \\
\hline IV & $1(4.20)$ & 0 & $6(18.8)$ & $3(4.2)$ & \\
\hline V & $16(66.7)$ & $9(56.2)$ & $14(43.8)$ & $43(62.3)$ & \\
\hline VI & $7(29.2)$ & $7(43.8)$ & $12(37.5)$ & $23(33.3)$ & \\
\hline
\end{tabular}

ple negative tumours [13]. In the study conducted by Youk et al., regardless of the PR status, in contrast to our study, they found the ADC values of triple negative tumours higher than Her 2-enriched tumours [1]. Rapid growth and developing intra-tumoural necrosis are responsible for higher ADC values [14].

The higher rate of rim enhancement in triple negative tumours was similar to previous data $[1,15]$. Overexpression of vascular endothelial growth factor and intratumoral hypoxia are the cause of rim enhancement, which is a malignancy indicator [15].

We found a type 2 enhancement curve more frequently in the triple negative group. Uematsu et al. found a type 1 curve to be more frequent in triple negative tumours [14], Angelini et al. found no difference in terms of enhancement curves and molecular subtypes [15]. Although the type 3 enhancement curve has been defined for malignant lesions, triple negative tumours can show type 1 and type 2 enhancement curves and may be confused with benign lesions. The contrast enhancement curve alone is misleading in distinguishing triple negative tumours from benign lesions, and other sequences should be carefully evaluated. Circumscribed margin is another misleading and confusing MRI feature of triple negative tumours. Uematsu et al. found circumscribed margin with MRI [14]; Wang et al. found same feature with mammography and ultrasound more frequently in TNBC [16].

The grade 3 tumour ratio was higher and the grade 1 tumour ratio was lower in the triple negative tumours. No grade 1 triple negative tumour was detected, as in the study by Chas et al. [17]. Type 2 and type 3 enhancement curves were more frequently detected in grades 2 and 3 tumours, and type 1 enhancement curve was more frequently detected in grade 1 tumours. This was compatible with previous data [18].

Lymph node involvement was most common in Her 2-enriched tumours and least common in luminal A tumours. Her 2-enriched tumours progress more aggressively compared to luminal A, and the risk of nodal and local recurrence after breast conserving surgery was found 
to be similar to triple negative tumours [19]. This explains the high rate of lymph node involvement in Her 2-enriched tumours in our study.

While the majority of malignant breast cancers were hypointense on T2W TSE images in the study of Kuhl et al., in our patients the tumours were most commonly isointense and least commonly hypointense on T2W images [20]. T2W hyperintensity is highly suggestive of TNBC due to intra-tumoural necrosis, but we found no significant difference between T2W intensity and molecular subtypes. Hasebe et al. suggested that the presence of fibrotic foci in necrotic circumscribed tumours may be an indicator of repairment, and the outcome may be different than without fibrotic foci [21]. In our study, the lack of difference between the groups in $\mathrm{T} 2 \mathrm{~W}$ images may be due to the presence of more fibrotic foci in TNBC tumours. This may indicate that there are different subtypes of triple negative tumours. Since there was no follow-up of the patients in our study, we cannot comment about the patient outcome and survey.

Multifocal lesions were observed in 33.3\% and multicentric lesions in $19.1 \%$ of the patients. In the study of Neri et al., the rate of multifocality was $11.3 \%$ and the rate of multicentricity was $5.2 \%$; these rates were very low compared to our study [22]. The reason for this discrepancy is that in the vast majority of patients with existing MRI, the indication for breast MRI is local advanced stage breast cancer, and the evaluation of the response of the tumour to the treatment before and after neoadjuvant chemotherapy.

As stated in the study of Choi et al., no relationship was found between tumour grade and ADC value in our study [23]. This may be due to TNBCs in the grade 3 group despite higher ADC values.
The retrospective nature of our study and the relatively low number of patients with triple negative tumours compared to luminal A and B, the lower number of invasive lobular and mixed infiltrative cancer compared to invasive ductal cancer, and no follow-up of patients were the limitations of our study. Another limitation of our study was the exclusion of the non-mass-forming lesions. Because these lesions grow with a remaining normal parenchyma and this normal parenchyma may lead to erroneous results in ADC measurements, we decided to exclude these cases. MRI finding of a significant part of patients with lobular cancer is non mass enhancement. Thus, this may lead to a lower number of patients with lobular cancer in our study and inclusion of the patients with lobular cancer into the BI-RADS 4 category.

\section{Conclusions}

Higher ADC values, rim enhancement, and circumscribed margin are the distinctive features of TNBC tumours. The distinctive MRI features of TNBC should be well known. While the typical features of the TNBC tumour on MRI are present, if the pathology report is compatible with a different subtype, the pathologist should be informed of the situation, and the pathological specimen should be re-evaluated. This is very important to plan the patient's treatment and may prevent unnecessary targeted therapies.

\section{Conflict of interest}

The authors report no conflict of interest.

\section{References}

1. Youk JH, Son EJ, Chung J, et al. Triple-negative invasive breast cancer on dynamic contrast-enhanced and diffusion-weighted MR imaging: comparison with other breast cancer subtypes. Eur Radiol 2012; 22: 1724-1734.

2. Yamamoto Y, Iwase H. Clinicopathological features and treatment strategy for triple-negative breast cancer. Int J Clin Oncol 2010; 15: 341-351.

3. De Ronde JJ, Hannemann J, Halfwerk H, et al. Concordance of clinical and molecular breast cancer subtyping in the context of preoperative chemotherapy response. Breast Cancer Res Treat 2010; 119: 119-126.

4. Denley H, Pinder SE, Elston CW, et al. Preoperative assessment of prognostic factors in breast cancer. J Clin Pathol 2001; 54: 20-24.

5. Montagna E, Bagnardi V, Rotmensz N, et al. Immunohistochemically defined subtypes and outcome in occult breast carcinoma with axillary presentation. Breast Cancer Res Treat 2011; 129: 867-875.

6. Martincich L, Deantoni V, Bertotto I, et al. Correlations between diffusion-weighted imaging and breast cancer biomarkers. Eur Radiol 2012; 22: 1519-1528.
7. Oakman C, Viale G, Di Leo A. Management of triple negative breast cancer. Breast 2010; 19: 312-321.

8. Dent R, Trudeau M, Pritchard KI, et al. Triple-negative breast cancer: clinicalfeatures and patterns of recurrence. Clin Cancer Res 2007; 13: 4429-4434.

9. Haffty BG, Yang Q, Reiss M, et al. Locoregionalrelapse and distantmetastasis inconservativelymanaged triple negative early-stage breast cancer. J Clin Oncol 2006; 24: 5652-5657.

10. Hammond ME, Hayes DF, Dowsett M, et al. American Society of Clinical Oncology/College of American Pathologists guideline recommendations for immunohistochemical testing of estrogen and progesterone receptors in breast cancer. J Clin Oncol 2010; 28: 27842795.

11. Heywang-Köbrunner SH, Bick U, Bradley WG Jr, et al. International investigation of breast MRI : results of a multicentre study (11 sites) concerning diagnostic parameters for contrast-enhanced MRI based on 519 histopathologically correlated lesions. Eur Radiol 2001; 11: 531-546. 
12. Horvath E, Bañuelos OR, Silva CF, et al. Triple receptor-negative breast cancer. How is it seen on imaging findings? Rev Chil Radiol 2012; 18: 97-106.

13. Xie T, Zhao Q, Fu C, et al. Differentiation of triple-negative breast cancer from other subtypes through whole-tumor histogram analysis on multiparametric MR imaging. Eur Radiol 2019; 29: 2535-2544.

14. Uematsu T, Kasami M, Yuen S. Triple-negative breast cancer: correlation between MR imaging and pathologic findings. Radiology 2009; 250: 638-647.

15. Angelini G, Marini C, Iacconi C, et al. Magnetic resonance (MR) features in triple negative breast cancer (TNBC) vs receptor positive cancer (nTNBC). Clin Imaging 2018; 49: 12-16.

16. Wang Y, Ikeda DM, Narasimhan B, et al. Estrogen receptor-negative invasive breast cancer: imaging features of tumors with and without human epidermal growth factor receptor type 2 overexpression. Radiology 2008; 246: 367-375.

17. Chas M, Boivin L, Arbion F, et al. Clinicopathologic predictors of lymph node metastasis in breast cancer patients according to molecular subtype. J Gynecol Obstet Hum Reprod 2018; 47: 9-15.
18. Bluemke DA, Gatsonis CA, Chen MH, et al. Magnetic resonance imaging of the breast prior to biopsy. JAMA 2004; 292: 2735-2742.

19. Kennecke H, Yerushalmi R, Woods R, et al. Metastatic behavior of breast cancer subtypes. J Clin Oncol 2010; 28: 3271-3277.

20. Kuhl CK, Klaschik S, Mielcarek P, et al. Do T2 weighted pulse sequences help with the differential diagnosis of enhancing lesions in dynamic breast MRI? J Magn Reson Imaging 1999; 9: 187-196.

21. Hasebe T, Tsuda H, Hirohashi S, et al. Fibrotic focus in infiltrating ductal carcinoma of the breast: a significant histopathological prognostic parameter for predicting the long-term survival of the patients. Breast Cancer Res Treat 1998; 49: 195-208.

22. Neri A, Marrelli D, Megha T, et al. Clinical significance of multifocal and multicentric breast cancers and choice of surgical treatment: a retrospective study on a series of 1158 cases. BMC Surg 2015; 15 : 1.

23. Choi SY, Chang YW, Park HJ, et al. Correlation of the apparent diffusion coefficiency values on diffusion-weighted imaging with prognostic factors for breast cancer. Br J Radiol 2012; 85: 474-479. 